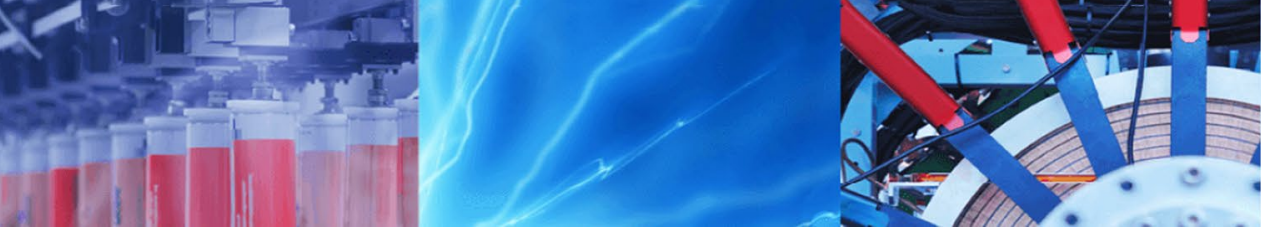

Research Article

\title{
Reverse micelle assisted hydrothermal reaction route for the synthesis of homogenous $\mathrm{MoS}_{2}$ nanospheres
}

\author{
J. S. Arya Nair ${ }^{1} \cdot$ R. Aswathi ${ }^{1} \cdot$ K. Y. Sandhya ${ }^{1}$ (D)
}

(c) Springer Nature Switzerland AG 2019

\begin{abstract}
Here in, for the first time, reverse micelle assisted relatively low temperature hydrothermal method has been reported to synthesize $\mathrm{MOS}_{2}$ nanospheres (NS). The formation of $\mathrm{MOS}_{2}$ NS was confirmed from TEM, SEM, XRD and EDAX analysis. Further, the influence of surfactant, stabilizing agent and temperature on the morphology, size and size distribution of the $\mathrm{MoS}_{2}$ NS has been discussed coherently.
\end{abstract}

Keywords Molybdenum disulphide $\cdot$ Nanospheres $\cdot$ Reverses micelle $\cdot$ Hydrothermal

\section{Introduction}

Molybdenum disulfide $\left(\mathrm{MoS}_{2}\right)$ is a two dimensional (2D) semiconductor material of the type $\mathrm{MX}_{2}$, comprising $\mathrm{X}-\mathrm{M}-\mathrm{X}$ units bonded covalently and held together by weak van der Waals force of attraction [1]. $\mathrm{MoS}_{2}$ nanostructures have gained tremendous attention in many advanced applications such as catalysis [2], photo electrochemical hydrogen evolution reactions (HER) [3], battery electrode materials [4], as electrochemical and fluorescence sensing materials [5], and as a photocatalyst [6] due to its multitude of diverse of properties such as large active specific surface area, flexibility, visible light absorbing ability and semiconductivity. The size and shape of the $\mathrm{MoS}_{2}$ nanostructures determine the extent of tunability of its exotic physical and chemical properties, and hence morphology selective and size exclusive synthesis methods have to be developed and adopted, for the different potential applications.

$\mathrm{MoS}_{2}$ nanostructures of various shapes such as 2D sheets [7], nanoparticles (NP) [8], nanospheres (NS) [9], nanorods [10], nanoflowers [11], and quantum dots [12] are gaining attention over the past decade and are prepared by methods such as exfoliation [13], chemical vapor deposition(CVD) [14], hydrothermal/solvothermal methods [15], gas-phase synthesis [16], sol-gel methods [17] etc. The synthesis of $\mathrm{MoS}_{2} \mathrm{NS}$ is generally carried out using hydro/solvothermal methods at high temperatures using various precursors in the presence of a reducing agent, for e.g. ammonium heptamolybdate tetrahydrate $\left[\left(\mathrm{NH}_{4}\right)_{6} \mathrm{Mo}_{7} \mathrm{O}_{24} \cdot 4 \mathrm{H}_{2} \mathrm{O}\right]$ and thiourea [18], $\left[\left(\mathrm{NH}_{4}\right)_{6} \mathrm{Mo}_{7} \mathrm{O}_{24} \cdot 4 \mathrm{H} 2 \mathrm{O}\right]$ and ammonium polysuphide [19], and $\left[\left(\mathrm{NH}_{4}\right)_{6} \mathrm{Mo}_{7} \mathrm{O}_{24} \cdot 4 \mathrm{H}_{2} \mathrm{O}\right]$ and disodium monosulfide in the presence $\mathrm{HCl}$ [9]. Upon close observation of the reported synthetic procedures, it can be noted that most are carried out at high temperatures which renders the structural stability, shape, and structure of the formed $\mathrm{MoS}_{2}$ detrimental to the potential use. And interestingly, the $\mathrm{MoS}_{2}$ NS previously reported is constituted of fragments of $\mathrm{MoS}_{2}$ sheets with irregular shape and with no well defined inherent structure. Further, it has been reported that as the temperature increases, the spherical structure of $\mathrm{MoS}_{2}$ transforms to polyhedral [20]. With this backdrop at the outset, we are reporting a novel facile, easy to scale-up lower temperature reverse-micelle

Electronic supplementary material The online version of this article (https://doi.org/10.1007/s42452-019-0528-y) contains supplementary material, which is available to authorized users.

K. Y. Sandhya, sandhya@iist.ac.in | 1 Department of Chemistry, Indian Institute of Space Science and Technology, Valiyamala, Thiruvananthapuram 695547, India.

SN Applied Sciences (2019) 1:508 | https://doi.org/10.1007/s42452-019-0528-y 
assisted hydrothermal synthesis method to obtain coherent $\mathrm{MoS}_{2} \mathrm{NS}$, using sodium molybdate and thioacetamide as the precursor and the reducing agent, respectively. Unlike the reported methods, the reaction was spatially confined to nanospaces created by reverse-micelles and this is the first time report on the synthesis of $\mathrm{MoS}_{2} \mathrm{NS}$ using reverse micelles. Further, the effect of synthetic conditions, such as temperature and the presence of surfactant and/or stabilizing agent on the size, size distribution and the shape of the NS were studied and the results duly discussed.

\section{Experimental}

\subsection{Materials}

The reagents thioacetamide $\left(\mathrm{C}_{2} \mathrm{H}_{5} \mathrm{NS}\right)$ and sodium molybdate dehydrate $\left(\mathrm{Na}_{2} \mathrm{MoO}_{4} \cdot 4 \mathrm{H}_{2} \mathrm{O}\right)$ are purchased from Sigma-Aldrich. Polyethylene glycol 200 [PEG] and TRITON $\mathrm{X}-100$ were obtained in liquid form from Merck Specialties Private Limited. All the reagents were used as procured.

\subsection{Synthesis of $\mathrm{MoS}_{2}$ nanospheres}

In a typical synthesis, $\mathrm{Na}_{2} \mathrm{MoO}_{4} \cdot 4 \mathrm{H}_{2} \mathrm{O}(68 \mathrm{mg})$ and of $\mathrm{C}_{2} \mathrm{H}_{5} \mathrm{NS}$ (42.26 mg) were dissolved in $3 \mathrm{~mL}$ of distilled water. To this PEG $(12 \mathrm{~mL})$ and TRITON X-100 $(20 \mu \mathrm{L})$ were added and sonicated for $30 \mathrm{~min}$. The solution was transferred to a $25 \mathrm{~mL}$ stainless steel Teflon-lined autoclave and kept for reaction at $120^{\circ} \mathrm{C}$ or $180^{\circ} \mathrm{C}$ for $24 \mathrm{~h}$. The resulting solution was cooled to room temperature. The black colored product formed was filtered and washed several times with distilled water and finally dried in a vacuum oven at $80^{\circ} \mathrm{C}$. Similarly the reaction was carried out in the absence of either of Triton X-100 or PEG or both to study the role played by the individuals in determining the size and morphology of the desired nanostructure.

\subsection{Characterization}

The morphology and the size of the samples were determined using high resolution transmission electron microscope (HR-TEM) and scanning electron microscope (SEM). Energy dispersive spectroscopy (EDS) was performed using (JEOL JEM 2100). UV-Visible spectrum was recorded using CARY 100 Bio UV-Visible spectrophotometer. Universal Attenuated Total Reflection (UATR) mode of transform infrared (FT-IR) spectroscopy was employed for recording IR spectra using Perkin Elmer spectrum 100 FT-IR spectrophotometer. Raman spectroscopy was done by using Renishaw confocal Raman microscope with a $530 \mathrm{~nm}$ laser. The phase analysis was performed through powder
X-ray diffraction (XRD, Bruker AXS D8 Advance using Cu Ka radiation $\left(\lambda=1.5406 A^{\circ}\right)$. The particle size analysis has been carried out with Zeta sizer Nano ZS Series, Malvern Instruments, Malvern, UK.

\section{Results and discussion}

The synthesis of $\mathrm{MoS}_{2}$ NS was attempted by lower temperature $\left(120 / 180^{\circ} \mathrm{C}\right)$ reverse micelle assisted hydrothermal route using sodium molybdate as the precursor. This bottom up approach does not use any harmful organic solvents to form the micelle and unlike that of the top down approaches, our method avoids the requirement of severe conditions or complicated post treatment processes. The hydrophilic precursor is spatially confined to occupy the nanospaces created by the hydrophobic ends of the reverse micelle and the reaction between the precursors and the reducing agent takes place inside the nanospaces hence formed to form the nano $\mathrm{MoS}_{2}$ spheres. The mechanism of the formation of the NS is illustrated in Scheme 1.

The HR-TEM images Fig. 1a-d shows the $\mathrm{MoS}_{2}$ nanostructure formed at the temperature of $120^{\circ} \mathrm{C}$ and $180^{\circ} \mathrm{C}$. The $\mathrm{MoS}_{2} \mathrm{NS}$ formed at $180^{\circ} \mathrm{C}$ possess a uniform spherical shape, smooth surface and are in the size range of 200-280 $\mathrm{nm}$ whereas those formed at $120^{\circ} \mathrm{C}$ has a size range of $125-130 \mathrm{~nm}$. The peaks corresponding to Mo and $S$, in the EDS spectrum (Fig. 1e) confirms the presence of $\mathrm{Mo}$ and $\mathrm{S}$ in the ratio of $0.5: 1$, respectively and agrees with the expected stoichiometry of $\mathrm{MoS}_{2}$. The relatively smooth surface and the spherical shape of the NS can be attributed to the reverse micelle assisted synthesis. The presence of PEG, which is used as the stabilizing agent, renders the use of additional organic solvents superfluous and unnecessary for dispersal of TRITON X-100, and also minimizes the aggregation of the NS, thereby enhancing the stability of the nanospheres. Further, the synthesis was conducted in the absence either the surfactant or stabilizing agent or both, in an attempt to confirm the reverse micelle assistance and to further understand the mechanism of formation. The SEM image (Fig. 2a) of the $\mathrm{MoS}_{2}$ in the absence of both the surfactant and the stabilizing agent yielded bulk and flaky $\mathrm{MoS}_{2}$ as opposed to the NS morphology expected which confirms the role of reverse micelle in the formation of the NS. The bulk $\mathrm{MoS}_{2}$ thus formed was not dispersible in water unlike their $\mathrm{MoS}_{2}$ NS counterparts formed in the presence of surfactant and stabilizing agents. Additionally, no Tyndall effect was observed as is demonstrated in Fig. 2b. However, in the absence of surfactant alone, i.e., solely in the presence of PEG, the formation of NS, though with higher sizes and distribution, was observed (Fig. 2c, d) and is explained in 


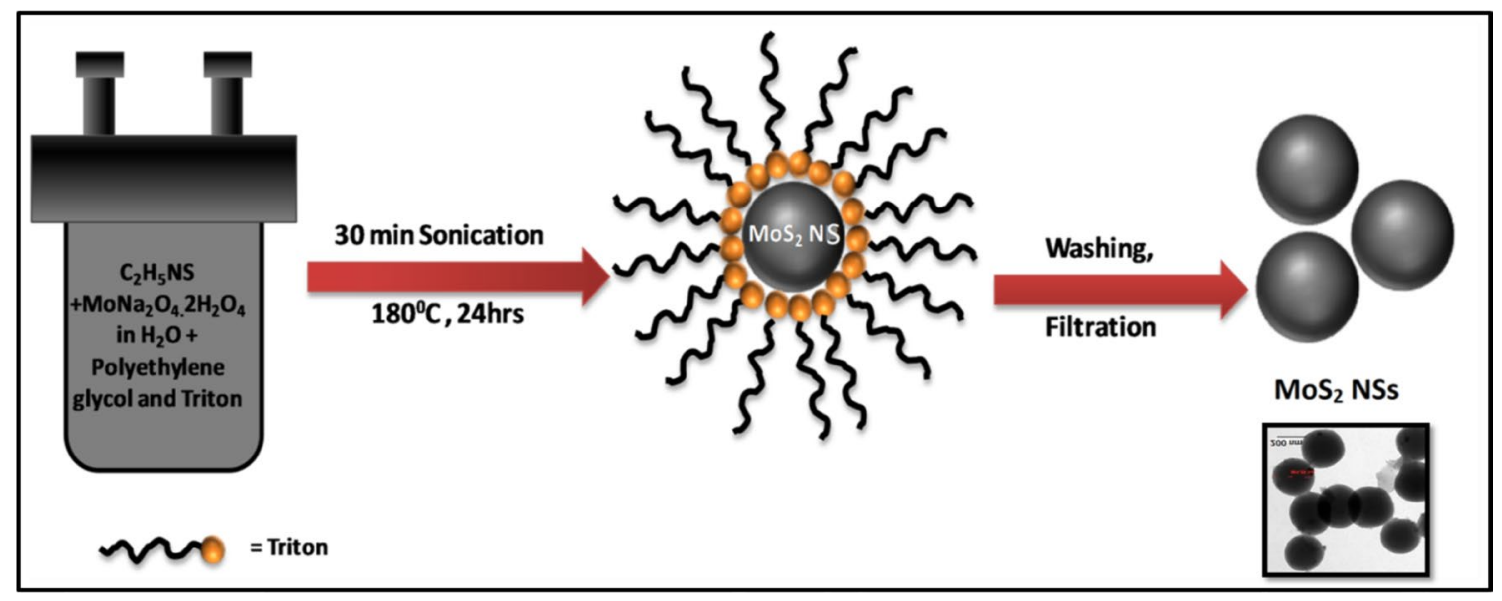

Scheme 1 Illustration of the synthesis and formation of $\mathrm{MoS}_{2} \mathrm{NS}$ by reverse micelle assisted hydrothermal route

Scheme 2. The $\mathrm{MoS}_{2}$ NS obtained was further characterized using UV-Vis, FTIR, Raman spectroscopic techniques and XRD.

The FTIR spectrum (Fig. 3a) of the $\mathrm{MoS}_{2}$ NS bears the characteristic peaks of $\mathrm{MoS}_{2}$ at -849 and $1089 \mathrm{~cm}^{-1}$ corresponding to the Mo-O vibrations [21]. The broad peak at $-3441 \mathrm{~cm}^{-1}$ is assigned to the $-\mathrm{OH}$ stretching of the water molecules intercalated between the $\mathrm{MoS}_{2}$ layers. Three Raman-active modes at 280,377, and $405 \mathrm{~cm}^{-1}$ were observed for the $\mathrm{MoS}_{2}$ NS in the Raman spectrum (Fig. 3b) and they correspond to the longitudinal acoustic phonon modes of $2 \mathrm{H}-\mathrm{MoS}_{2}$. The sharp peak around $280 \mathrm{~cm}^{-1}$ is due to the $\mathrm{E}_{1 \mathrm{~g}}$ mode and the peaks at -377 and $405 \mathrm{~cm}^{-1}$ are due to the in-plane $E_{2 g}^{1}(\mathrm{~S}-\mathrm{Mo}-\mathrm{S})$ and out-of-plane $\mathrm{A}_{1 \mathrm{~g}}$ $(\mathrm{S}-\mathrm{S})$ mode, respectively. Normally the frequency for $E_{2 g}^{1}$ mode occurs near $383 \mathrm{~cm}^{-1}$ and the shift in the peak may be due to a stronger dielectric screening of the long range columbic interaction in the $\mathrm{MoS}_{2}$ layers as the separation between the periodically repeated layers increases. It is known that the Raman mode spacing between $E_{2 g}^{1}$ and $A_{1 g}$ provides information about the layer thickness of $\mathrm{MoS}_{2}$. The frequencies of the corresponding modes are expected to be indicative of the number of layers present, that is, as the number of layers increases the spacing between the two modes also increases. For bulk $\mathrm{MoS}_{2}$ the spacing between these two modes is $-56 \mathrm{~cm}^{-1}$ and for monolayer, it is $-19 \mathrm{~cm}^{-1}$. The observed shift in the $M_{0}{ }_{2} N S$ is $28 \mathrm{~cm}^{-1}$ which suggests that the $\mathrm{MoS}_{2} \mathrm{NS}$ are possibly made of few layered sheets, as the value leans closer to that of the monolayer but far lesser than that observed in bulk. The additional peaks around 335 and $350 \mathrm{~cm}^{-1}$ may be due to the anomalous behavior of $E_{2 g}^{1}$ mode while this anomalous frequency trend possibly arises due to the (i) interactions other than Van der Waals forces, (ii) relative displacement between Mo and S atoms and/or (iii) due to additional long-range Coulomb interactions [22] each of which can be attributed to the spherical shape of the $\mathrm{MoS}_{2}$ which imparts strain and hence relative displacement of the Mo and $\mathrm{S}$ atoms. Raman studies can be further used to confirm the presence of 1T phase of $\mathrm{MoS}_{2}$ which has peaks at 150, 225 and $325 \mathrm{~cm}^{-1}$ corresponding to the $J_{1}, J_{2}$ and $J_{3}$ mode of vibration, respectively. The absence of these peaks in the Raman spectrum of the $\mathrm{MoS}_{2}$ NS thereby indicates the absence of the metallic $1 \mathrm{~T}-\mathrm{MoS}_{2}$ phase while asserting the presence of semiconductor $2 \mathrm{H}$ phase [23].

The optical properties of the $\mathrm{MoS}_{-}$NS were studied using the UV-Vis spectrophotometer and is given in Fig. 3c. Usually, the bulk $\mathrm{MoS}_{2}$ possesses two prominent absorption bands around 620 and $680 \mathrm{~nm}$ due to the $B$ and $A$ excitons, respectively, arising from the $k$ points of the Brillouin zone. In the prepared $\mathrm{MoS}_{2} \mathrm{NS}$, these peaks are strongly blue shifted and the bands are observed near 280 and $370 \mathrm{~nm}$ wavelengths, possibly attributed to the quantum confinement effect. Though the size of the $\mathrm{MoS}_{2}$ NS is in the range of $250-300 \mathrm{~nm}$, the quantum effect is observed and hence is possibly due to the few layered sheets which form the NS [24] which augments the conclusion drawn from Raman analysis of the $\mathrm{MoS}_{2} \mathrm{NS}$.

Figure 3d shows the XRD pattern of $\mathrm{MoS}_{2} \mathrm{NS}$ and $\mathrm{MoS}_{2}$ bulk, all the diffraction peaks of bulk MoS $\mathrm{S}_{2}$ can be easily indexed to the hexagonal phase (JCPDS No. 37-1492). MoS $_{2}$ exhibits peaks at 14.1, 32.7, 36.7, 39.0, $45.0,49.9,57.0,58.56$ and 60.5 which can be specifically assigned to the (002), (100), (102), (103), (006), (105), (106), (110) and (008) planes and in comparison, $\mathrm{MoS}_{2}$ NS though basically retains the position of most of the diffraction peaks of $\mathrm{MoS}_{2}$ (denoted by \# symbol). The most important XRD feature which provides a proof for the existence of the hexagonal unit cells of $\mathrm{MoS}_{2}$ is the observation of the diffraction peaks due to (002) planes [25]. The peaks at $2 \theta$ of $19.23^{\circ}$ and $23.34^{\circ}$ are possibly 
Fig. 1 a-d TEM images of synthesized $\mathrm{MoS}_{2} \mathrm{NS}$ at $180^{\circ} \mathrm{C}$ and $120^{\circ} \mathrm{C} \mathbf{e}$ is the EDS spectrum of the $\mathrm{MoS}_{2} \mathrm{NS}$ at $180^{\circ} \mathrm{C}$


due to the presence of PEG (denoted by * symbol) which could be acting as the capping agent and hence leading to the non-aggregated state of the $\mathrm{MoS}_{2} \mathrm{NS}$. The absence of any other peaks confirms the formation and the purity of the $\mathrm{MoS}_{2}$ NS. The reasonably sharp peaks in the XRD spectrum are indicative of the crystalline nature of the formed NS.

\section{Effect of temperature and surfactant on the Size of $\mathrm{MoS}_{2} \mathrm{NS}$}

To understand the effect of temperature on the NS, the synthesis was carried out at a lower temperature $\left(120^{\circ} \mathrm{C}\right)$ and was characterized using various techniques (Fig. 3). 
Fig. 2 The SEM images and Tyndall effect experiment of a and $\mathbf{b}$ the $\mathrm{MoS}_{2}$ formed in the absence of TRITON and PEG, which shows bulk $\mathrm{MoS}_{2}$ and confirms the absence of $\mathrm{MoS}_{2}$ NS, $\mathbf{c}$ and $\mathbf{d} \mathrm{MoS}_{2}$ NS formed in the presence of PEG(alone) and the Tyndall effect observed for its dispersion in water

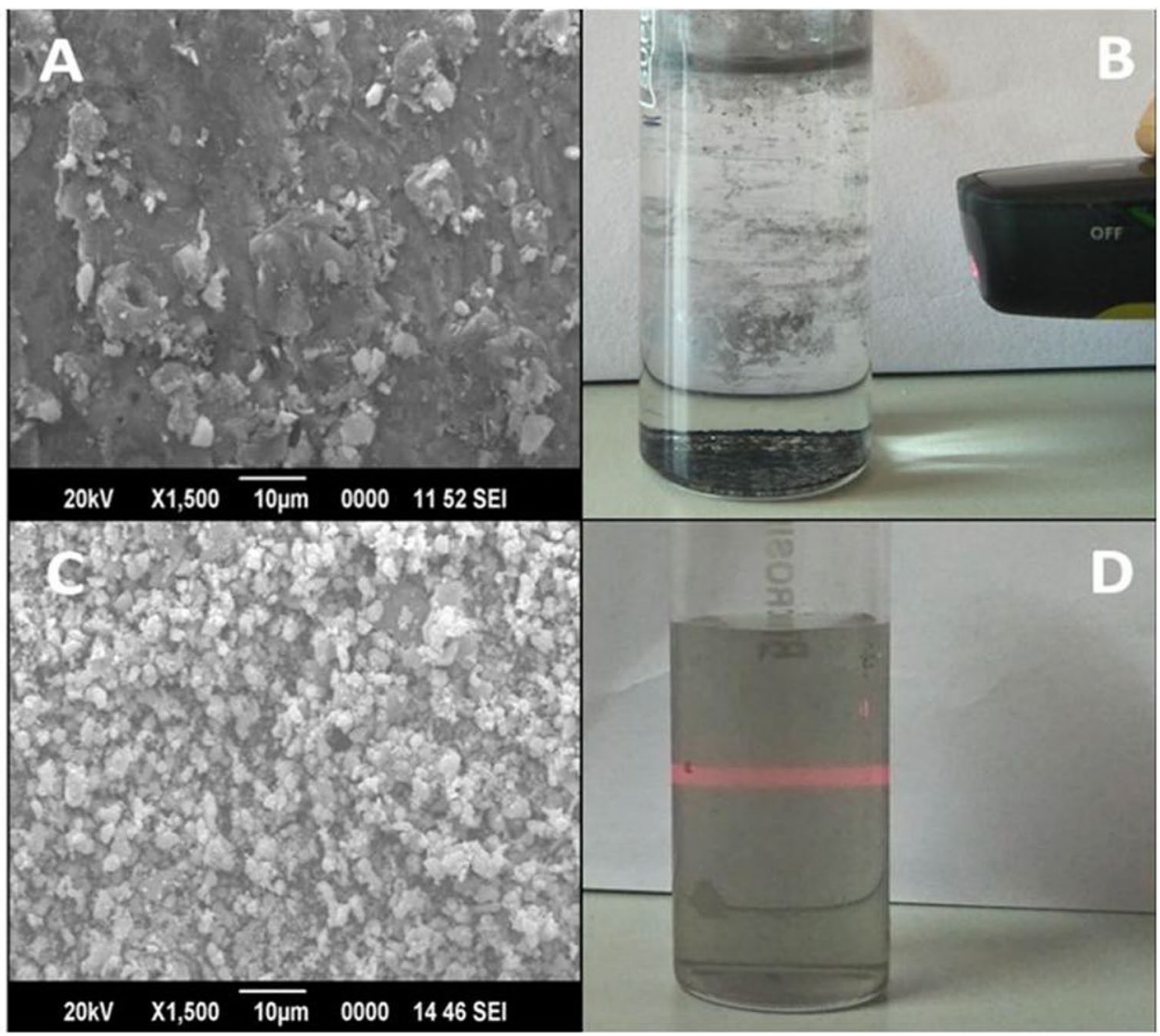

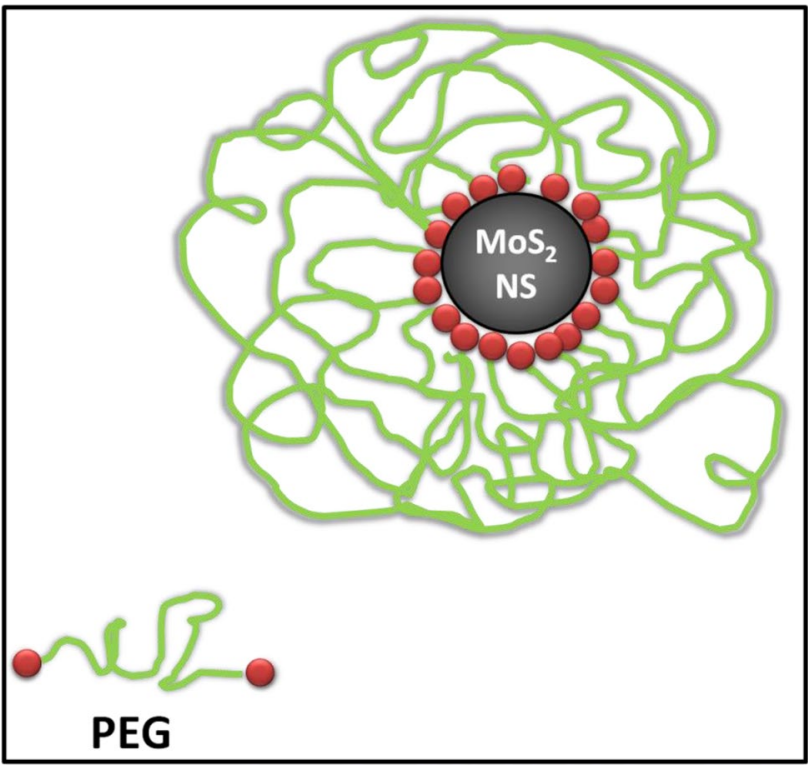

Scheme 2 Illustration of the inverse micelle formation by PEG, the entanglement of the long chains and the heads on both the sides makes the inverse micelle sizes non-uniform at lower temperatures
The result shows that the formation of NS occurs even at a temperature as low as $120^{\circ} \mathrm{C}$ and was confirmed by TEM images (Fig. 1C, d) and Tyndall effect (Fig. S1). The effect of temperature on the size of the $\mathrm{MoS}_{2}$ NS was investigated by particle size analyzer and the results are shown in Fig S2 and S3. Compared to that of the average size of NS formed at $180^{\circ} \mathrm{C}$, which was $-250 \mathrm{~nm}$ (Fig. $\mathrm{S} 2 \mathrm{~A}$ ), the size of the NS at $120^{\circ} \mathrm{C}$ (Fig. S3A) was lower in size $(-130 \mathrm{~nm})$ which is supported by the TEM images (Fig. 1c). The result shows that the size of the NS can be reduced by lowering the temperature while keeping the reaction time fixed. The reduction in size can be attributed to the lower reaction rate at $120^{\circ} \mathrm{C}$ compared to that of at $180^{\circ} \mathrm{C}$. Moreover, as the temperature increases, there is a possibility that two or three micelles merging to form a bigger sized micelle [26] which might also serve as the reason for the increase in the size of NS.

Further to study the effect of surfactant to the morphology, size and size distribution, the synthesis was conducted in the absence of surfactant, (i.e., in the presence of PEG alone) at both the temperatures $\left(120\right.$ and $\left.180^{\circ} \mathrm{C}\right)$. The formation of NS was observed even in the absence of the surfactant as suggested by SEM analysis (Fig. 2C), Tyndall effect (Fig. 2d) and the DLS analysis (S2B and S3B). The sizes of NS in its absence were higher with 580 and 

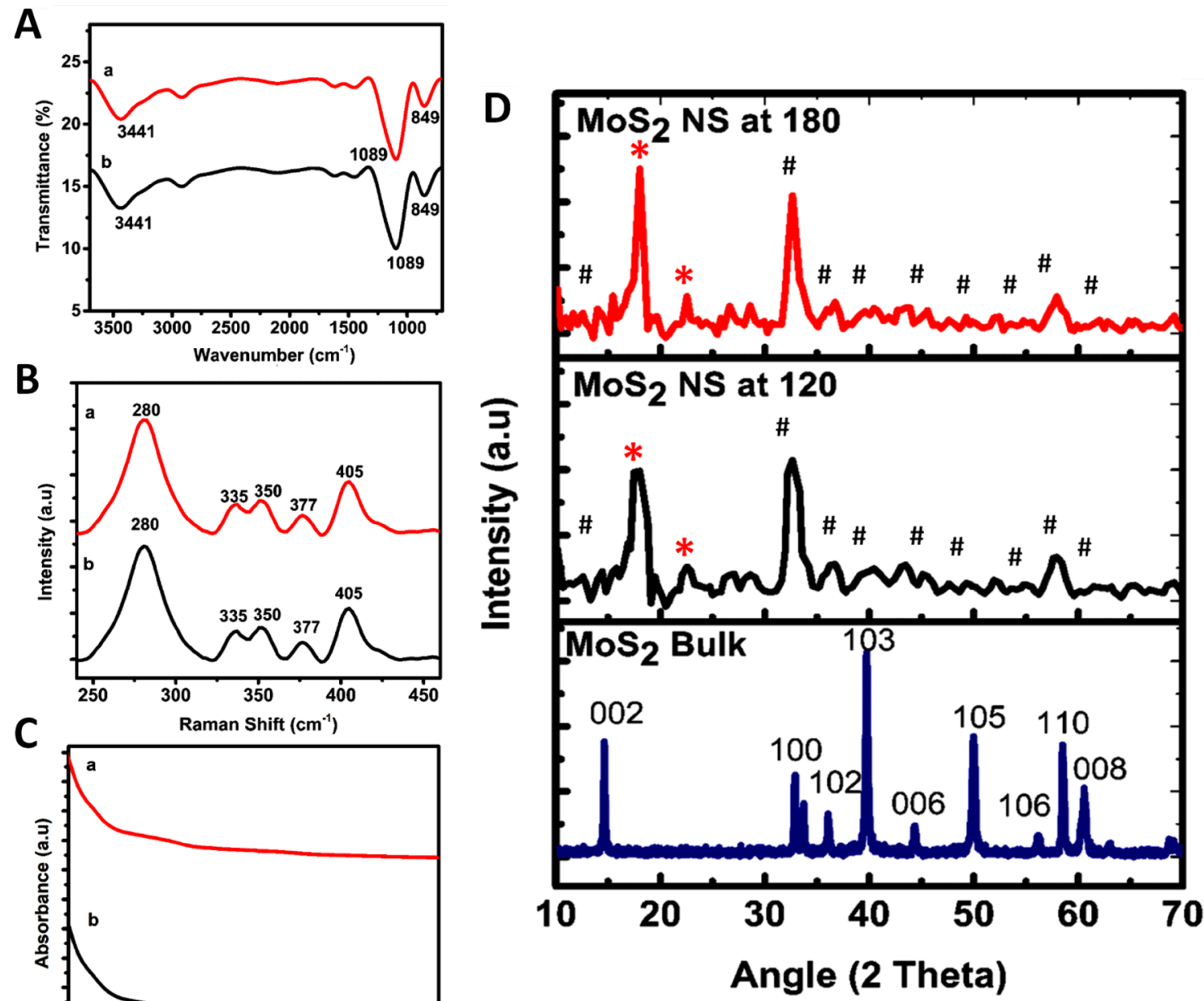

Fig. 3 The characterization results of the $\mathrm{MoS}_{2}$ NS formed at 180 (a) and $120{ }^{\circ} \mathrm{C}$ (b): a FT-IR spectrum b Raman spectrum c UV-Visible absorption spectrum and $\mathbf{d}$ the XRD pattern

$370 \mathrm{~nm}$ at 120 and $180^{\circ} \mathrm{C}$ compared to that of in the presence of the surfactant ( 130 and $250 \mathrm{~nm}$ ), respectively, and the change in the size was more pronounced at the lower temperature $\left(120^{\circ} \mathrm{C}\right)$. It is interesting to note that in the presence of surfactant the size was higher at higher temperature. This result suggests that at the lower temperature $\left(120^{\circ} \mathrm{C}\right)$ the stabilizing agent was not able to form uniform sized reverse micelle thus resulting in the wider distribution of NS, owing to the sluggishness of the bulky and entangled PEG chains which finds difficult to arrange in an uniform way at lower temperature compared to that of at higher temperature as illustrated in Scheme 2. The formation of NS in the presence of PEG alone at both temperatures can invoke an explanation citing its amphiphilic nature which aids information of the reverse micelle.

From the above observations, it is clear that $\mathrm{MoS}_{2} \mathrm{NS}$ is formed at the temperature as low as $120^{\circ} \mathrm{C}$ by this method and the results confirm the role of the reverse micelle in the formation of smooth surfaced and regular NS. Further, it is observed that though a stabilizing agent, PEG, itself is capable of aiding the formation of $\mathrm{MoS}_{2} \mathrm{NS}$, a narrow/uniform size distribution of NS necessitates a higher temperature. These results indicate that the size and size distribution of the MoS NS can be altered by tuning the synthetic conditions and thus this approach can be reliably adopted for synthesizing different sizes of $\mathrm{MoS}_{2}$ NS.

\section{Conclusion}

In this work, we have demonstrated a novel and simple reverse micelle assisted lower temperature hydrothermal method for smooth surfaced and well-shaped $\mathrm{MoS}_{2}$ NS. The average diameters of $\mathrm{MoS}_{2}$ NS were -125 and $280 \mathrm{~nm}$ 
at 120 and $180^{\circ} \mathrm{C}$, respectively. The usage of various structural and morphological characterization tools confirmed the formation and purity of the obtained $\mathrm{MoS}_{2}$ NS. The influence of temperature and surfactant on the morphology, size and size distribution of the product was studied and has been subsequently inferred that the presence of both surfactant and stabilizing agent at low temperatures are essential requisites for obtaining $\mathrm{MoS}_{2}$ NS with well defined shape and size. We have hereby detailed a versatile novel method which can further be widely adapted to obtain a plethora of $\mathrm{MoS}_{2}$ nanostructures.

Acknowledgements Financial support from Indian Institute of Space Science and Technology (IIST), Trivandrum is greatly acknowledged.

\section{References}

1. Geim AK, Grigorieva IV (2013) Van der Waals heterostructures. Nature 499:419

2. Chen J, Li S-L, Xu Q, Tanaka K (2002) Synthesis of open-ended $\mathrm{MoS}_{2}$ nanotubes and the application as the catalyst of methanation. Chem Commun 16:1722-1723

3. Jinlong L, Wenli G, Tongxiang L, Ken S, Hideo M (2017) Improving hydrogen evolution reaction for $\mathrm{MoS}_{2}$ hollow spheres. J Electroanal Chem 799(Supplement C):304-307

4. Stephenson T, Li Z, Olsen B, Mitlin D (2014) Lithium ion battery applications of molybdenum disulfide $\left(\mathrm{MoS}_{2}\right)$ nanocomposites. Energy Environ Sci 7(1):209-231

5. Xu Y-L, Niu X-Y, Chen H-L, Zhao S-G, Chen X-G (2017) Switch-on fluorescence sensor for ascorbic acid detection based on $\mathrm{MoS}_{2}$ quantum dots $-\mathrm{MnO}_{2}$ nanosheets system and its application in fruit samples. Chin Chem Lett 28(2):338-344

6. Li Y, Li Y-L, Araujo CM, Luo W, Ahuja R (2013) Single-layer MoS, as an efficient photocatalyst. Catal Sci Technol 3(9):2214-2220

7. Lee $\mathrm{Y}-\mathrm{H}$, Zhang $\mathrm{X}-\mathrm{Q}$, Zhang W, Chang $\mathrm{M}-\mathrm{T}$, Lin C-T, Chang K-D, Yu Y-C, Wang JT-W, Chang C-S, Li L-J, Lin T-W (2012) Synthesis of large-area $\mathrm{MoS}_{2}$ atomic layers with chemical vapor deposition. Adv Mater 24(17):2320-2325

8. Santillo G, Deorsola FA, Bensaid S, Russo N, Fino D (2012) MoS nanoparticle precipitation in turbulent micromixers. Chem Eng J 207-208:322-328

9. Tian Y, Zhao X, Shen L, Meng F, Tang L, Deng Y, Wang Z (2006) Synthesis of amorphous $\mathrm{MoS}_{2}$ nanospheres by hydrothermal reaction. Mater Lett 60(4):527-529

10. Lin H, Chen X, Li H, Yang M, Qi Y (2010) Hydrothermal synthesis and characterization of $\mathrm{MoS}_{2}$ nanorods. Mater Lett 64(15):1748-1750

11. Wang D, Pan Z, Wu Z, Wang Z, Liu Z (2014) Hydrothermal synthesis of $\mathrm{MoS}_{2}$ nanoflowers as highly efficient hydrogen evolution reaction catalysts. J Power Sour 264:229-234

12. Gu W, Yan Y, Zhang C, Ding C, Xian Y (2016) One-step synthesis of water-soluble $\mathrm{MoS}_{2}$ quantum dots via a hydrothermal method as a fluorescent probe for hyaluronidase detection. ACS Appl Mater Interfaces 8(18):11272-11279

13. Thripuranthaka M, Chandra Sekhar R, Dattatray JL (2014) MoS nanoparticles and $\mathrm{h}-\mathrm{BN}$ nanosheets from direct exfoliation of bulk powder: one-step synthesis method. Mater Res Express 1(3):035038

14. Dhar S, Kranthi Kumar V, Choudhury TH, Shivashankar SA Raghavan S (2016) Chemical vapor deposition of MoS layers from Mo-S-C-O-H system: thermodynamic modeling and validation. Phys Chem Chem Phys 18(22):14918-14926

15. Sun Y, Wang S, Wang Q (2009) Flowerlike $\mathrm{MoS}_{2}$ nanoparticles: solvothermal synthesis and characterization. Front Chem China 4(2):173-176

16. Zak A, Feldman Y, Alperovich V, Rosentsveig R, Tenne R (2000) Growth mechanism of $\mathrm{MoS}_{2}$ fullerene-like nanoparticles by gasphase synthesis. J Am Chem Soc 122(45):11108-11116

17. Guo X, Wang Z, Zhu W, Yang H (2017) The novel and facile preparation of multilayer $\mathrm{MoS}_{2}$ crystals by a chelation-assisted solgel method and their electrochemical performance. RSC Adv 7(15):9009-9014

18. Li C, Li J, Wang Z, Zhang S, Wei G, Zhang J, Wang H, An C (2017) The synthesis of hollow $\mathrm{MoS}_{2}$ nanospheres assembled by ultrathin nanosheets for an enhanced energy storage performance. Inorg Chem Front 4(2):309-314

19. Vattikuti SVP (2015) Synthesis and structural characterization of $\mathrm{MoS}_{2}$ nanospheres and nanosheets using solvothermal method. J Nanomater 50(14):5024-5038

20. Hu K, Hu X (2009) Formation, exfoliation and restacking of $\mathrm{MoS}_{2}$ nanostructures. Mater Sci Technol 25(3):407-414

21. Ganganagappa N, Tharamani C, Chandrappa G, Livage J (2007) Hydrothermal synthesis of amorphous $\mathrm{MoS}_{2}$ nanofiber bundles via acidification of ammonium heptamolybdate tetrahydrate. Nanoscale Res Lett 2(9):461

22. Li H, Zhang Q, Yap CCR, Tay BK, Edwin THT, Olivier A, Baillargeat D (2012) From bulk to monolayer $\mathrm{MoS}_{2}$ : evolution of Raman scattering. Adv Funct Mater 22(7):1385-1390

23. Yin $X$, Wang $Q$, Cao L, Tang CS, Luo X, Zheng Y, Wong LM, Wang SJ, Quek SY, Zhang W, Rusydi A, Wee ATS (2017) Tunable inverted gap in monolayer quasi-metallic MoS2 induced by strong charge-lattice coupling. Nat Commun 8(1):486

24. Pallikkarathodi Mani N, Ganiga M, Cyriac J (2017) Synthesis of $\mathrm{MoS}_{2}$ quantum dots uniformly dispersed on low dimensional $\mathrm{MoS}_{2}$ nanosheets and unravelling its multiple emissive states. ChemistrySelect 2(21):5942-5949

25. Zeng Y-X, Zhong X-W, Liu Z-Q, Chen S, Li N (2013) Preparation and enhancement of thermal conductivity of heat transfer oil-based $\mathrm{MoS}_{2}$ nanofluids. J Nanomater. https://doi. org/10.1155/2013/270490

26. Muller N (1993) Temperature dependence of critical micelle concentrations and heat capacities of micellization for ionic surfactants. Langmuir 9(1):96-100

Publisher's Note Springer Nature remains neutral with regard to jurisdictional claims in published maps and institutional affiliations. 